\title{
Robust Optimization of System Compliance for Physical Interaction in Uncertain Scenarios
}

\author{
G. M. Gasparri ${ }^{\dagger}$, F. Fabiani ${ }^{\dagger}$, M. Garabini ${ }^{\dagger}$, L. Pallottino ${ }^{\dagger}$, M. Catalano ${ }^{\dagger \ddagger}$, G. Grioli $^{\dagger \dagger}$, R. Persichini $^{\dagger}$ \\ and A. Bicchi ${ }^{\dagger \dagger}$
}

\begin{abstract}
Compliance in robot design and control is often introduced to improve the robot performance in tasks where interaction with environment or human is required. However a rigorous method to choose the correct level of compliance is still not available. In this work we use robust optimization as a tool to select the optimal compliance value in a robotenvironment interaction scenario under uncertainties. We propose an approach that can be profitably applied on a variety of tasks, e.g.manipulation tasks or locomotion tasks. The aim is to minimize the forces of interaction considering model constraints and uncertainties. Numerical results show that: i) in case of perfect knowledge of the environment stiff robots behave better in terms of force minimization, ii) in case of uncertainties the optimal stiffness of the robot is lower than the previous case and optimal solutions provide a faster task accomplishment, iii) the optimal stiffness decreases as a function of the uncertainty measure. Experiments are carried out in a realistic set-up in case of bi-manual object handover.
\end{abstract}

\section{INTRODUCTION}

In recent years the robotics research community has significantly turned its attention towards Soft Robotics [1] as a response to the urgent need for robots that can safely interact with living beings and effectively operate in unstructured natural, or humanized, environments.

The key idea in soft robots is the introduction of fixed or adjustable compliant elements in the robot design. Among the several concepts that has been presented in the literature, two are the most common: Series Elastic Actuators (SEA) [2] and Variable Impedance Actuators (VIA) (see [3] for a recent review). Soft robots show better performances w.r.t. their rigid counterpart in several aspects. From higher robustness against unpredictable impacts [4], to faster operations under safety constraints [5], to the possibility to store and release energy into the springs (as in [6] and [7]) for energy efficiency, to finish with the ability to double the peak speed of a conventional motor (see e.g. [8] and [9]).

The recent results suggest that Soft Robotics provides new opportunities in the task accomplishment where highly dynamic motions and safe interactions are required as in case of manipulation and locomotion tasks. The physical interaction is a common event that can be observed in multidisciplinary fields e.g: robot-humans interaction in medical applications or industrial co-working frameworks, robot-robot interaction

\footnotetext{
*This work was supported by the European Commission projects (FP7 framework) Walk-Man and the European Commission Grant no. H2020ICT-645599 "SOMA": SOft MAnipulation

$\dagger$ Centro Int. di Ricerca "E. Piaggio", Univ. of Pisa, 56126 Pisa, Italy

$\doteqdot$ Department of Advanced Robotics, Istituto Italiano di Tecnologia, Genoa, Italy
}

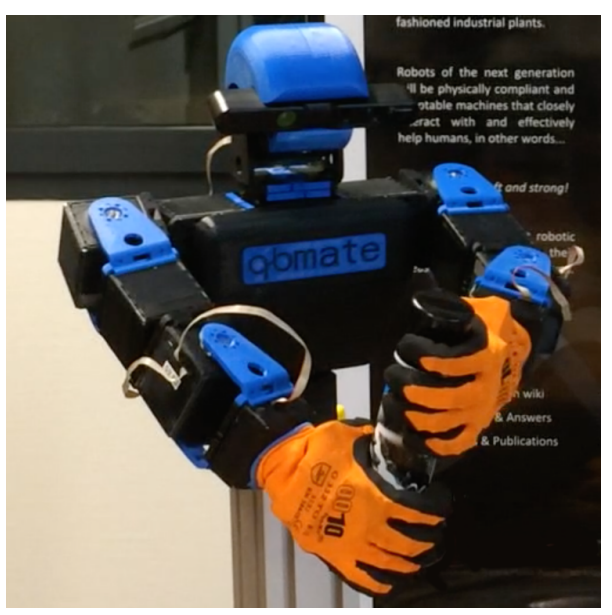

Fig. 1. A bi-manual Soft Robot while performing an handover.

for bimanual operations in industrial manufacturing or robotenvironment interactions in the robot workspace.

The planning and control strategies must be designed to take into account the possible occurrence of both scheduled and unexpected interactions and uncertainties in the scenario or model uncertainties. In the literature there are many examples ([10], [11] to cite a few) claiming that soft is better when robots must operate in unknown environment, nevertheless, at the best of our knowledge, a solid formalism supporting this claim has not yet been provided, nor a method to choose the proper stiffness value.

This paper presents a method to choose, in scenarios with uncertainties, the optimal stiffness of the robot to accomplish a desired task minimizing the interaction forces (i.e., force exchanged between two entities). This definition of the problem can then be specialized depending on which entity interacts with the robot: human-robot interaction, environment-robot interaction, robot-robot interaction etc. The goal of this paper is to move forward in the direction of providing methodology for the determination of the system compliance in interaction tasks. The Robust Optimization theory [12] is here used to solve a case study problem and, based on a careful analysis of results, to provide basic principles to be adopted in more realistic scenarios. Robust Optimization (RO) has been widely used in several fields where the presence of unmodeled uncertainties may greatly influence the success of the global task/mission (e.g. [13], [14]). In particular, the importance of optimization robotic pick and place operations has been recently emphasized 
in [15], where RO is treated as an important extension to classical global optimization. In [16] the authors adopt the RO in order to compute a dynamically feasible timeparametrized trajectory for robot manipulators along a fixed geometric path with contact constraints.

The considered problem is first translated into a robust optimization problem, which, due to the linear property of the model considered, can be recast into two deterministic convex optimization problems. Case study of this work is the handover manipulation task for a bi-manual manipulator as the one depicted in Fig. 1. An object in an uncertain position, has to be exchanged between two end effectors and the robot stiffness has to be set in order to minimize the exchanged forces. After a careful analysis of the simulative and experimental results the following lessons can be learned:

- If the framework is perfectly known the rigid system performs best, while in case of uncertainty the optimal stiffness for executing the interaction task is roughly proportional to the inverse of the uncertainty.

- in the handover task the optimal stiffness of the two end-effectors to minimize the interaction forces has to be in a stiff-soft configuration.

- the usage of a compliant configuration soft-soft of the end-effectors does not provide optimal solutions neither in term of execution time nor in term of minimum exchanged forces.

The results and the method of this work are potentially useful and will be exploited in several scenarios, by improving:

- the robustness of walking robots in rough terrain where the compliance of the foot can be adjusted to minimize the reaction forces due to a not perfect knowledge of the ground height;

- the interaction in the environment where the uncertainty is not limited to the position but regards also parameters of the environment (e.g., stiffness and damping);

- the physical human-robot interaction in which the uncertainty is not limited to model parameters but can include also the intention of the human agent, with application in assistive devices.

\section{Problem Statement}

In this section we consider the dynamics of two manipulators accomplishing the handover task. A linear model is provided in order to have a treatable problem on which robust optimization can be applied. Let $\Omega_{1}$ e $\Omega_{2}$ be the dynamics of two soft manipulators actuated by the Variable Stiffness Actuators, e.g. Qbmove [17]. The mutual effects of the manipulators' interactions can be modeled as external forces acting on the end effectors (namely e.e. in the paper). Let $n$ be the number of the actuated joints, $n_{p_{i}}$ the number of the contact points where interaction occurs (i.e. on the e.e). The dynamics $\Omega_{1}$ (and similarly $\Omega_{2}$ ) is:

$$
\begin{aligned}
& M(q) \ddot{q}+N(q, \dot{q})=h(q-\theta, \sigma)+B(\dot{q}-\dot{\theta})+\sum_{i=1}^{n_{p_{i}}} J_{i} \omega_{\text {ext }} \\
& I \ddot{\theta}+D \dot{\theta}+h(q-\theta, \sigma)+B(\dot{q}-\dot{\theta})=\tau_{c}
\end{aligned}
$$

where $q \in \mathbb{R}^{n}$ is the link position vector, $\theta \in \mathbb{R}^{n}$ is the motor position vector, $M(q) \in \mathbb{R}^{n x n}$ is the inertia matrix of the manipulator, $N(q, \dot{q}) \in \mathbb{R}^{n}$ is the vector of the centrifugal/Coriolis and gravity contribution and $B \in \mathbb{R}^{n x n}$ is the matrix of the viscous friction in the joints. The matrix $h(q-\theta, \sigma)$ represents the non linear elastic characteristic of the VSAs which depends on the deflection $\delta=q-\theta$ of the actuator elastic element and on the stiffness reference $\sigma . I \in \mathbb{R}^{n x n}$ and $D \in \mathbb{R}^{n x n}$ are the actuation inertia and the damping matrices respectively. $\omega_{\text {ext }_{i}} \in \mathbb{R}^{6 x 1}$ is the $i-t h$ external wrench which acts on the robot in the $p_{i} \in \mathbb{R}^{6 x 1}$ point of contact whose geometric Jacobian matrix is $J_{i} \in \mathbb{R}^{6 x n}$. Finally, $\tau_{c}$ is the control input. For the handover task we assume that both systems are in contact with the handled object during the task execution. Moreover, e.e. are considered jointed to the object. Let $m$ be the payload mass, $\ddot{\zeta}$ its acceleration and $\omega$ the wrench exerted on it, both expressed in the object inertial frame $\{L\}$. The payload dynamics is $m \ddot{\zeta}=\omega$ where $\omega=\sum_{i=1}^{2}{ }^{L} G_{i} \omega_{i}$ and ${ }^{L} G_{i}$ is the grasp matrix which maps the exerted manipulator wrench $\omega_{i}$ into the equivalent e.e. wrench $\omega$. Let ${ }^{L} J_{A_{i}}$ be the analytical Jacobian matrices of the $i$-th manipulator. The coupled dynamics of manipulators and payload in the operative space w.r.t $L$ is:

$$
\left(m+\sum_{i=1}^{2}{ }^{L} M_{A_{i}}\right) \ddot{\zeta}=\sum_{i=1}^{2}{ }^{L} N_{A_{i}} \dot{\zeta}+\sum_{i=1}^{2}{ }^{L} \omega_{A_{i}}+{ }^{L} \omega_{m}
$$

Let $M_{A_{i}}, N_{A_{i}}, \omega_{A_{i}}=T_{A}^{T} \omega_{i}$ be the equivalent inertia matrix, coriolis/gravity contribution and wrench of $\Omega_{i}$ respectively (for $i=1,2$ ) and $T_{A}^{T}$ be the transformation matrix between the geometric and analytical jacobians (for more details see [18]). Let $\zeta$ be the mass displacement and $\dot{\zeta}, \ddot{\zeta}$ its derivatives (and hence the e.e. displacement and derivatives respectively). Finally ${ }^{L} \omega_{m}$ is an external wrench applied on the mass $m$ which models the payload transition from one e.e. to the other. The aim of this work is to optimize both manipulators compliance and controls minimizing the exchanged forces during the handover task in presence of uncertainties. The resulting optimization problem is non linear and non convex and it is not reported here for space limitations. Based on the aforementioned assumptions on the system during the task execution, it is possible to define the optimization problem on a linearized model. In order to optimize both compliance and controls, the optimization problem is then solved obtaining the optimal controls for a given constant compliance. The optimal compliance is finally obtained based on the evaluation of the exchanged forces for different compliance values.

Let $\Sigma$ be a generic Multi Input Multi Output Linear Time Invariant system on which a constant external force acts. Defining $x \in \mathbf{R}^{n_{s}}$ the $n_{s}$-dimensional state vector, and $u \in \mathbf{R}^{n_{i}}$ the $n_{i}$-dimensional input control vector, the $\Sigma$ state space dynamics is:

$$
\dot{x}=A x+B u+T, \quad F=H x+G u,
$$

where $A$ is the state space matrix, $B$ is the input matrix and $T$ the external forces vector. Let $F$ be the vector of measurable interaction forces that is linear in the state and the control, and it is represented by matrices $H$ and $G$. Let $\left[t_{0}, t_{f}\right]$ be the 


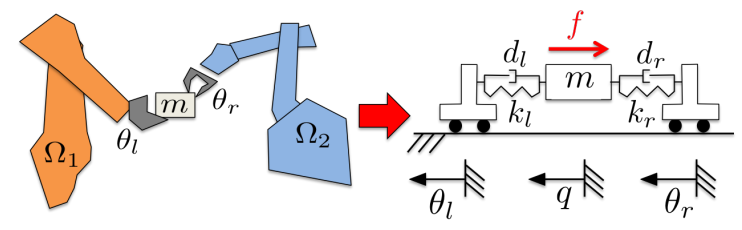

Fig. 2. Scheme of the interacting manipulators. The equivalent linear model of the end effectors and mass is depicted on the right.

time interval for the task execution, $x_{0}$ and $x_{f}$ be the initial and terminal states respectively, $\left[x_{\min }, x_{\max }\right]$ be the minimum and maximum limits of $x$. Let $\left[u_{\min }, u_{\max }\right]$ be the minimum and maximum limits of $u$. In this paper we consider uncertainties on the initial state of the system representing for example a partially known position of the object. Indeed, let $X_{0}$ be the space of the uncertain initial states. The goal is to find a control input $u(t)$ that minimizes the interaction forces, verifying initial and terminal conditions and state and control constraints. The optimal control problem is hence:

$$
\begin{aligned}
\underset{u(t)}{\operatorname{Minimize}} & \frac{1}{2} \int_{t_{0}}^{t_{f}} F^{2}(t) d t \\
\text { s.t. } & \dot{x}(t)=A x(t)+B u(t)+T \\
& x\left(t_{0}\right)=x_{0} \in X_{0} \\
& x\left(t_{f}\right)=x_{f} \\
& x_{\min } \leq x(t) \leq x_{\max } \\
& u_{\min } \leq u(t) \leq u_{\max } .
\end{aligned}
$$

A scheme of the systems considered for the case study is depicted in Fig. 2. The object payload $m$ that has to be transfered from one e.e. to the other can be modeled as a further external force $f$ acting on one of the manipulators. Hence, at the terminal instant of the task, to model the occurred object exchange the force has to be completely exerted by the other manipulator. The solutions obtained on the linear model are then applied to nonlinear complex systems and validated experimentally.

\section{PROBLEM SOLUTION}

In this section, the optimal control problem is transformed into an optimization one that is thus extended to take into account uncertainties in the initial conditions. The so obtained robust optimization problem is finally decoupled into two deterministic and convex optimization ones in which the uncertainty is at its bounds. It will be also shown that, infeasibility of one of the two deterministic problems implies the infeasibilty of the robust optimization. On the other hand, the worst performing deterministic solution (between the two of them) is the solution also of the robust optimization problem.

1) Discretization: from Optimal Control to Optimization Problems: The first step in the solution process is to follow the classical approach of discretizing the continuous dynamics of the system and transforming the optimal control problem into an optimization one. The discretized dynamics is

$$
x(i+1)=A_{d} x(i)+B_{d} u(i)+T_{d}
$$

where the matrices $A_{d}, B_{d}$, and $T_{d}$ are obtained from the continuous ones via standard discretization methods (e.g., Zero-Order-Hold or Tustin). Let $T_{S}$ be the sample time, $N$ be the number of the intervals in which the time $t=\left[t_{0}, t_{f}\right]$ is partitioned and, finally, let $i \in\{0, \ldots, N\}$ be the $i-t h$ time interval $\left[t_{i}, t_{i+1}\right]$ or, equivalently, the $i$-th step of the discrete system evolution. The discretized robust optimization problem is

$$
\begin{aligned}
\min _{U} & \frac{1}{2}\left\|F_{T}\right\|_{2}^{2} \\
\text { subject to } & A_{\text {in }} U \leq b_{\text {in }} \\
& A_{\text {eq }} U=b_{e q} \\
& u_{\min } S \leq U \leq u_{\max } S \\
& \forall x_{0} \in \mathbb{X}_{0}
\end{aligned}
$$

where $\mathbb{X}_{0}$ is the numerable and not empty set of possible initial states due to uncertainties on one or more components. In the rest of this section all other variables in (3) will be computed in terms of the dynamic matrices and interaction forces matrices $H$ and $G$. The vector $F_{T}=[F(0) F(1) \ldots F(N-1)]^{T}$ represents the total force exchanged during task execution, i.e.:

$$
F(i)=H x(i)+G u(i-1)
$$

with the state evolution given by

$$
x(i)=R_{i} U_{(i-1: 0)}+a_{i},
$$

where $R_{i}=\left[B_{d} A_{d} B_{d} A_{d}^{2} B_{d} \ldots A_{d}^{i-1} B_{d}\right] \in \mathbb{R}^{n_{s} \times i}$ is the controllability matrix at the $i$-th step, $U_{(i-1: 0)}=$ $\left[u(i-1)^{T} u(i-2)^{T} \ldots u(0)^{T}\right]^{T} \in \mathbb{R}^{i \times n_{i}}$ is the control vector from the initial to the $i-1$-th time step and $a_{i}=A_{d}^{i} x_{0}+\sum_{j=0}^{i-1} A_{d}^{j} T_{d} \in \mathbb{R}^{n_{s} \times 1}$ for $i \geq 1$. Finally, $S$ is a suitable selection matrix to bound the whole control vector $U=U_{(N-1: 0)}$. Replacing (5) in (4), we obtain that the vector $F_{T}$ is an affine function of both $U$ and $x_{0}$, i.e., $F_{T}=C U+\bar{H} d$, where:

$$
\begin{aligned}
& C=\left[\begin{array}{c}
E_{0} \\
E_{1} \\
\vdots \\
E_{N-1}
\end{array}\right] \in \mathbb{R}^{N \times N}, \quad \bar{H}=\left[\begin{array}{ccc}
H & & 0 \\
& \ddots & \\
0 & & H
\end{array}\right] \in \mathbb{R}^{N \times n s \times N} \\
& E_{i}=\left[0^{1 \times(N-i)}\left|G+H R_{i}(:, 1)\right| H R_{i}(:, 2: i-1)\right] \in \mathbb{R}^{n_{i} \times N} \\
& d=\underbrace{\left[\begin{array}{c}
I \\
A_{d} \\
A_{d}^{2} \\
\vdots \\
A_{d}^{N-1}
\end{array}\right]}_{d_{A}} x_{0}+\underbrace{\left[\begin{array}{c}
0 \\
T_{d} \\
A_{d} T_{d}+T_{d} \\
\vdots \\
\sum_{j=0}^{N-2} A_{d}^{j} T_{d}
\end{array}\right]}_{d_{T}}=d_{A} x_{0}+d_{T} \in \mathbb{R}^{n_{s} \times N \times 1},
\end{aligned}
$$

and $R_{i}(:, 1)$ is the first column of the controllability matrix at the $i$-th step, while $R_{i}(:, 2: i-1)$ are the remaining $i-$ 1 columns. The equality constraints of the problem (3) are given by the final condition $x(N)=x_{f}$, i.e.:

$$
x_{f}=R_{N} U+a_{N} \rightarrow R_{N} U=x_{f}-a_{N} \rightarrow A_{e q} U=b_{e q}
$$


On the other hand, the inequality constraints are consequences of the bounds on the state and on the control variables that depends on the particular model and the task characteristics. For this reason, inequality constraints will be explicitly computed in the next Section for the particular task scenario considered in this paper.

2) From Robust Optimization to Deterministic Problem: Consider the case of bounded uncertainties on the whole initial state $x_{0}$ or on one of its components. Without loss of generality and for the sake of clarity we suppose that uncertainty acts only on the $j$-th component $x_{0, j}$, i.e., we have that $\left|x_{0, j}\right| \leq \varepsilon$. Hence, in case of uncertainties, the whole initial state vector $x_{0}$ lays in $\mathbb{X}_{0}=\left\{x_{0}:\left|x_{0, j}\right| \leq \varepsilon\right\}$. The total force vector $F_{T}$ can be made explicitly dependent on the uncertain variable $x_{0, j}$ as follows:

$$
\begin{array}{r}
F_{T}=C U+\bar{H}\left(\tilde{d}+h x_{0, j}\right), \\
\text { with: } \tilde{d}=d_{A_{/ j}} x_{0}^{*}+d_{T} \quad \text { and } h=d_{A_{j}},
\end{array}
$$

where $x_{0}^{*}$ is the initial state vector with the $j-t h$ component null, i.e. $x_{0, k}^{*}=x_{0}$ for $k \neq j$ and $x_{0, j}^{*}=0 . d_{A_{j}}$ is the $j-t h$ column of the matrix $d_{A}$, while $d_{A / j}$ consists of the remaining $N-1$ columns of $d_{A}$. The functional in (6) is affine in the uncertain parameter $x_{0, j}$ (which belongs to a convex set); thus, applying classical results of the robust optimization theory [12], the worst case index cost for problem (3) leads to the minmax problem:

$$
\begin{aligned}
\min _{U} \max _{\left|x_{0, j}\right| \leq \varepsilon} & \frac{1}{2}\left\|C U+\bar{H} \tilde{d}+\bar{H} h x_{0, j}\right\|_{2}^{2} \\
\text { s.t. } & A_{i n} U \leq b_{i n}\left(x_{0, j}\right) \\
& A_{e q} U=b_{e q}\left(x_{0, j}\right) \\
& u_{\min } S \leq U \leq u_{\max } S .
\end{aligned}
$$

It is worth noting that the right hand side elements of inequality and equality constraints, $b_{i n}\left(x_{0, j}\right)$ and $b_{e q}\left(x_{0, j}\right)$, do linearly depend on the uncertain variable $x_{0, j}$ as it will be shown later in the paper. Hence, due to the convexity properties of the optimization problem, if one of the two subproblems (7) with $x_{0, j}= \pm \varepsilon$ is unfeasible so it is the robust optimization problem in (3). Conversely, if the subproblems are both feasible, then the problem in (3) is feasible for any $\left|x_{0, j}\right| \leq \varepsilon$. Moreover, if the minmax problem (7) is feasible, since the performance index is convex w.r.t. the bounded maximization variable $x_{0, j}$, it can be recast in the following form:

$$
\begin{gathered}
\max \{\psi(\varepsilon), \psi(-\varepsilon)\}, \\
\psi\left(x_{0, j}\right)=\min _{U} \frac{1}{2}\left\|C U+\bar{H} \tilde{d}+\bar{H} h x_{0, j}\right\|_{2}^{2} \\
\text { s.t. } \quad A_{\text {in }} U \leq b_{\text {in }}\left(x_{0, j}\right) \\
A_{e q} U=b_{e q}\left(x_{0, j}\right) \\
u_{\min } \underline{1} \leq U \leq u_{\max } \underline{1} .
\end{gathered}
$$

Concluding, it is worth noting that the Optimal Control problem under uncertainties in (2) can be tackled by solving the deterministic convex optimization problem (8) for the two boundary values of the uncertainty. Although general, in next sections the aforementioned approach is then formalized for the particular case under study, i.e. the handover task.
Moreover, an evaluation of the effects of different system compliances on the total force exchange will be proposed.

\section{HANDOVER TASK}

As case study we analyze an handover task in which an object, at an uncertain position determined through, e.g., vision, has to be passed from one e.e. to the other. In [19] the human-human handover has been extensively studied (neglecting uncertainties), and several performance indexes have been tested to evaluate the task. Conclusions are that the load transfer rate (force per time unit) can be used as quality index of the handover task. The same index will thus be used to evaluate the performance also in case of uncertainties. A planar linear model of the task can be obtained considering the logical scheme depicted in Fig. 3. Hence, the handover dynamics equation is:

$m \ddot{q}=-f-\left(q-\theta_{l}\right) k_{l}-\left(q-\theta_{r}\right) k_{r}-d_{l}\left(\dot{q}-\dot{\theta}_{l}\right)-d_{r}\left(\dot{q}-\dot{\theta}_{r}\right)$.

Let $\theta_{l}$ and $\theta_{r}$ be the equilibrium (or reference) positions of the end-effectors in contact with the object of mass $m$, whose position is denoted by $q$. Let the stiffness $k_{i}$ and the damping $d_{i}$ be the viscoelastic characteristic of the endeffectors. Finally, $f$ is the actual constant force that must be transferred from an end-effector to the other and represents the load to be handed over.

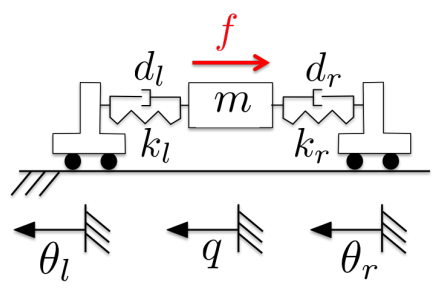

Fig. 3. The two carts and mass planar model. Each cart is in contact with the mass through a spring-damper system (the subscripts $r$ and $l$ stand for right and left, respectively). A constant force (in red) is assumed acting over the whole system representing the load to be transfered.

Defining the vector state $x=\left[q \dot{q} \theta_{l} \theta_{r}\right]^{T} \in \mathbb{R}^{4 \times 1}$ and the control input vector $u=\left[\dot{\theta}_{l} \dot{\theta}_{l}\right]^{T} \in \mathbb{R}^{2 \times 1}$, the state space dynamics becomes:

$$
\dot{x}=\underbrace{\left[\begin{array}{cccc}
0 & 1 & 0 & 0 \\
-\frac{\left(k_{l}+k_{r}\right)}{m} & -\frac{\left(d_{l}+d_{r}\right)}{m} & \frac{k_{l}}{m} & \frac{k_{r}}{m} \\
0 & 0 & 0 & 0 \\
0 & 0 & 0 & 0
\end{array}\right]}_{A} x+\underbrace{\left[\begin{array}{cc}
0 & 0 \\
\frac{d_{l}}{m} & \frac{d_{r}}{m} \\
1 & 0 \\
0 & 1
\end{array}\right]}_{B} u+\underbrace{\left[\begin{array}{c}
0 \\
-\frac{f}{m} \\
0 \\
0
\end{array}\right]}_{T} .
$$

The interaction forces, that the end effectors exert on the object, are given by the equation:

$$
F=\underbrace{\left[\begin{array}{cccc}
k_{l} & d_{l} & -k_{l} & 0 \\
k_{r} & d_{r} & 0 & -k_{r}
\end{array}\right]}_{H} x+\underbrace{\left[\begin{array}{cc}
-d_{l} & 0 \\
0 & -d_{r}
\end{array}\right]}_{G} u .
$$

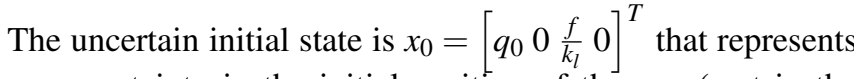
an uncertainty in the initial position of the e.e. (cart in the scheme) that handles the load. The terminal state requires that the load is eventually supported by the approaching 
e.e. and it can be represented as $x_{f}=\left[\begin{array}{llll}0 & 0 & 0 & \frac{f}{k_{r}}\end{array}\right]^{T}$. During the evolution, constraint on the position of the mass $m$ and bounds on the control inputs are taken into account to prevent unrealistic motions. Hence, considering the subproblem (8), the inequality constraints are consequences of the bounds on the state and on the control variables. Indeed, the displacement of the mass can be limited imposing:

$q_{\min } \leq e_{1}^{T} x(i) \leq q_{\max }$, with $e_{1}=\left[\begin{array}{llll}1 & 0 & 0 & 0\end{array}\right]^{T} \forall i=0, \ldots, N-1$.

where $q_{\min }$ and $q_{\max }$ are the minimum and maximum values of $q$ respectively. In matrix form:

$$
\underbrace{\left[\begin{array}{c}
e_{1}^{T} P_{0} \\
\vdots \\
e_{1}^{T} P_{N-1} \\
-e_{1}^{T} P_{0} \\
\vdots \\
-e_{1}^{T} P_{N-1}
\end{array}\right]}_{A_{\text {in }} \in \mathbb{R}^{N \times 2 N}} U \leq \underbrace{\left[\begin{array}{c}
q_{\max }-e_{1}^{T} a_{0} \\
\vdots \\
q_{\max }-e_{1}^{T} a_{N-1} \\
e_{1}^{T} a_{0}-q_{\min } \\
\vdots \\
e_{1}^{T} a_{N-1}-q_{\min }
\end{array}\right]}_{b_{\text {in }} \in \mathbb{R}^{N \times 1}},
$$

with $P_{i}=\left[\underline{0} \mid R_{i}\right], \underline{0} \in \mathbb{R}^{n_{s} \times 2(N-i)}$ and $n_{s}=4$. As anticipated, in case of uncertainties on the object's initial position we have that $\left|q_{0}\right| \leq \varepsilon$, hence the whole initial state vector $x_{0} \in$ $\mathbb{X}_{0}=\left\{\left[q_{0} 0 \frac{f}{k_{l}} 0\right]^{T}:\left|q_{0}\right| \leq \varepsilon\right\}$. The total force vector $F_{T}$ can be made explicitly dependent on $q_{0}$ as follows:

$$
\begin{gathered}
F_{T}=C U+\bar{H}\left(\tilde{d}+h q_{0}\right), \\
\text { with: } \tilde{d}=d_{A / 1}\left[\begin{array}{c}
0 \\
0 \\
\frac{f}{k_{l}} \\
0
\end{array}\right]+d_{T} \text { and } h=d_{A_{1}} \text {. }
\end{gathered}
$$

\section{Simulation Results}

In this section we provide a detailed discussion on the simulation results obtained by solving the problem (8) of system in Fig. 3 for different stiffness values and different terminal time.

Numerical values used for the simulations have been obtained based on the experimental set-up used for the experiments (see Fig. 7). In particular, the mass is $1[K g]$, sample time $T_{s}$ is $0.02[s]$ and the external force $f$ is $10[N]$. Problem (8) has been solved with elastic constants values within $[10,500][\mathrm{N} / \mathrm{m}]$, and a damping constant of $0.1[\mathrm{Ns} / \mathrm{m}]$ for both carts. Furthermore, the constraint on the maximum displacement of the mass is symmetrically set to $0.1[\mathrm{~m}]$, with the bound $\varepsilon$ on $q_{0}$ equal to $0.04[\mathrm{~m}]$ and the maximum velocity of each cart is fixed to $1[\mathrm{~m} / \mathrm{s}]$.

In Fig. 4, the minimum $N$ to find a solution of the optimization problem under uncertainties is reported for stiffness values in the range $(10,500)[N / m]$. Based on the simulation results, when at least one spring is very compliant (less than $50[N / m])$, the system requires many control steps $(N>50)$ to accomplish the handover task in presence of uncertainties. On the other hand, if the purpose is to minimize $N$ to accomplish the task, the optimal choice of having a low stiffness $(190.53[\mathrm{~N} / \mathrm{m}])$ the approaching e.e. and a high stiffness $(500[N / m])$ for the e.e. holding the mass. A further analysis has been performed by solving the optimal control problem fixing the terminal time large enough to ensure the problem feasibility for the entire stiffness range. The goal of the simulations analysis is to highlight the evolution of the optimal stiffness of the system in function of the uncertainties. In Fig. 5 the cost functional value $\left\|F_{\text {tot }}\right\|_{2}$ is depicted for a range of elastic constants for the nominal case and a case with uncertainty: $\varepsilon=0[\mathrm{~m}]$ and $\varepsilon=0.04[\mathrm{~m}]$. Referring to Fig. 5(a), if the initial position of the mass is accurately known (i.e. the uncertainty is zero $\varepsilon=0[m]$ ), the forces acting on the carts are minimized when each spring is maximally stiff (both $k_{l}$ and $k_{r}$ are set to $500[\mathrm{~N} / \mathrm{m}]$ ). Referring to Fig. 5(b), in case of maximum $0.04 \mathrm{~m}$ uncertainty on the initial position, the minimum internal forces are obtained with a more compliant approaching e.e. $\left(k_{l}=242.11[\mathrm{~N} / \mathrm{m}]\right.$ and $k_{r}=500[N / m]$ ). Figures 6(a) and 6(b), show the trend of the optimal compliance set and the performance index values w.r.t. the level of uncertainty, respectively. Obtained results confirm that whenever uncertainty on $q_{0}$ increases, the corresponding optimal cost $\left\|F_{\text {tot }}\right\|_{2}$ increases so as the optimal compliance of the left spring (i.e., stiffness decreases).

\section{EXPERIMENTAL TESTS}

In the following we describe the experimental setup and tests we have performed to validate the simulative results.

a) Experimental Setup: The experimental setup, depicted in Fig. 7 is composed of two planar 3-DoFs robotic arms (numbers 10 and 11 in the figure) actuated by variable stiffness actuators (Qbmove Maker Pro and Advanced [17], [20]). Each arm has a Pisa/IIT SoftHand [21] as end-effector (n.12 and n.13). The object (n.4) for the handover is mounted on a low-friction linear guide (n.7) and it is provided with two handles with Phidgets Micro Load Cells (n.5 and n.3), with supported load range $0-20[K g]$, for measuring the contact forces, and a custom position sensor (n.6) based on the Austrian Microsystem AS5040 that retrieves the position of the object. Two electronic boards (n.8 and n.9) are used for the acquisition of the force and position data. The external force $f$ is realized through a proper $0.5[K g]$ mass (n.2) connected to the object via a wire-pulley (n.1) system.

b) Experimental Tests: In the experimental tests we reproduced the handover task with three different configurations of the e.e. Cartesian stiffness set of left and right handles respectively: stiff-stiff, soft-soft and the optimal stiff-soft one. The handover operation has been implemented as follows. At the beginning the right hand grasps the right handle while supporting the external load. In order to reproduce the effects of the uncertainties the left arm is used to push (without grasping) the left handle generating an internal force between handles. Indeed, in the model depicted in Fig. 3, initial state uncertainty provides a variation in the initial value of the contact forces $F$. As soon as the left hand grasps the handle, the optimal references are commanded to the manipulators performing the handover. The simulations provide position and stiffness references for 


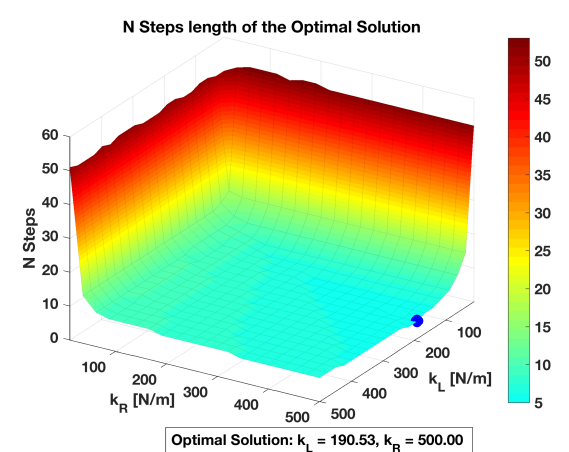

(a) 3D plot of stiffnesses w.r.t. numbers of steps $N$

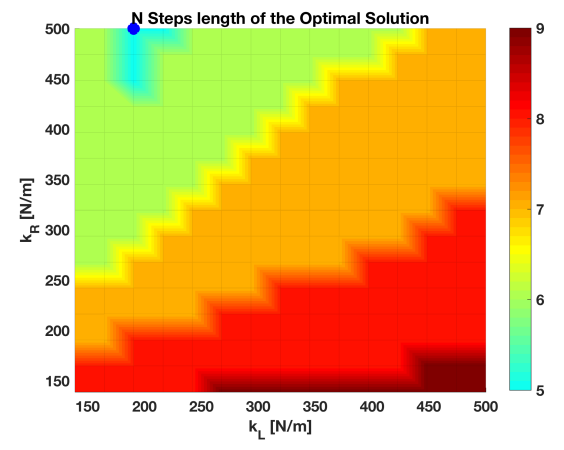

(b) Zoom on the plane of stiffnesses $k_{l}$ and $k_{r}$

Fig. 4. The plots depict the number of steps $N$ needed to optimally accomplish the handover task. The minimum time required in order to obtain a physical solution that minimize the internal forces $F$ is $N=5$ control steps and it corresponds to the pair of optimal stiffness $(190.53,500)[N / m]$.

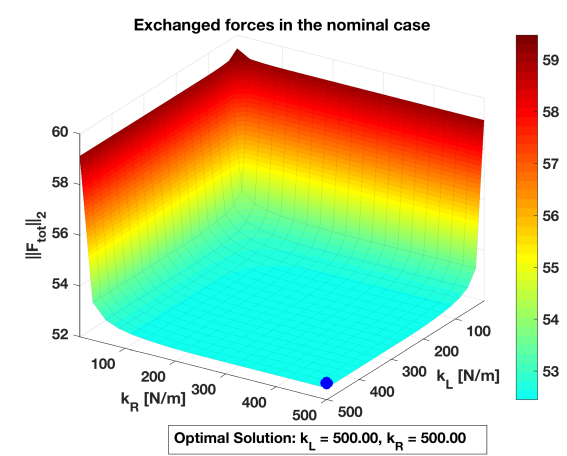

(a) Optimal stiffnesses for $N=52$ in case of $\varepsilon=0 \mathrm{~m}$

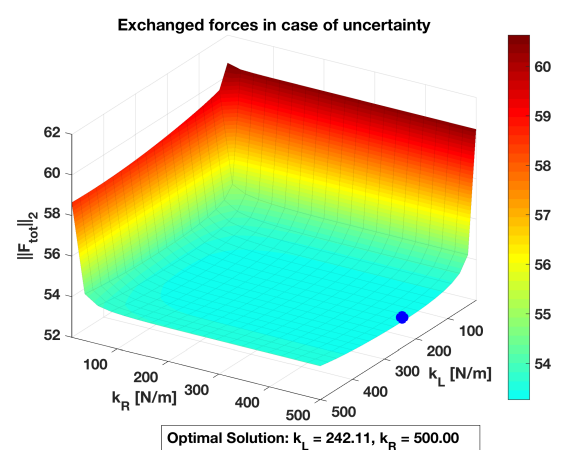

(b) Optimal stiffnesses for $N=52$ in case of $\varepsilon=$ $0.04 \mathrm{~m}$

Fig. 5. Optimal value of the cost functional $\left\|F_{\text {tot }}\right\|_{2}$ is reported for all possible combinations of the elastic constants within $[10,500][N / m]$ for a fixed number of $N=52$. The blue point denotes the minimum value of the function. When everything is known (Fig. $5(\mathrm{a})$ ), $k_{l}=k_{r}=k_{\max }=500$ are the optimal values of the system stiffnesses. On the other hand, in case of uncertainties (Fig. 5(b)), the optimal is obtained for a stiffness $\left(k_{l}\right)$ of the approaching cart lower than the stiffness $\left(k_{r}\right)$ of the cart holding the load.

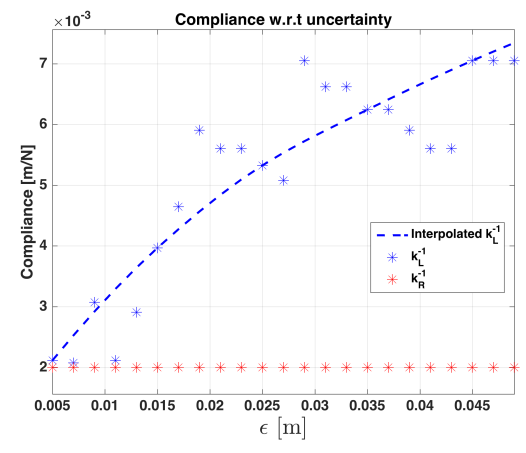

(a) Optimal compliance of left cart as a function of the uncertainty

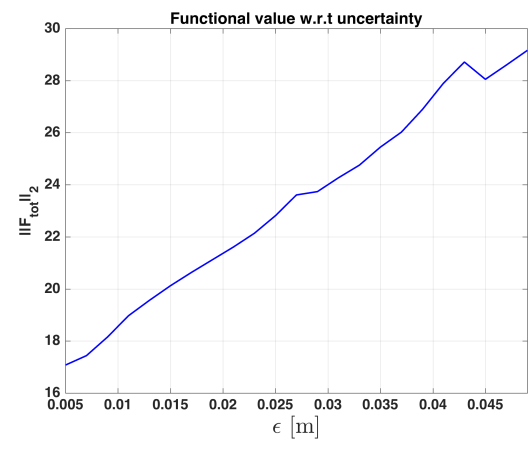

(b) Optimal cost function values

Fig. 6. In 6(a) the evolution of the optimal compliance of the approaching cart $1 / k_{l}$ of the system for $\varepsilon \in[0 \mathrm{~m}, 0.05 \mathrm{~m}]$ with a fixed low compliance $1 / 500 \mathrm{~N} / \mathrm{m}$ of the other cart is shown. The corresponding optimal cost value is reported in $6(\mathrm{~b})$.

the experiments. In particular, $\theta_{i}(t)$ are used as references for the Cartesian equilibrium positions of the arms. On the other hand, the stiffnesses $k_{L}$ and $k_{R}$ are used as Cartesian stiffness references at the end-effectors. Such stiffnesses must first be translated into joints' stiffness. In the following, the procedure to map the desired Cartesian stiffness matrix into joint one is described. Given $J(q)$ the Jacobian matrix of a manipulator in configuration $q$ and the Cartesian stiffness matrix $K_{C}$, the joint stiffness matrix $K_{J}$ is:

$$
K_{J}=J(q)^{T} K_{C} J(q)+\frac{\partial J(q)^{T}}{\partial q} K_{C} \Delta_{x},
$$

where $\Delta x$ is the Cartesian displacement between the desired and the actual position. Given a desired Cartesian stiffness matrix $\hat{K}_{C}$, following the approach proposed in [22], a diagonal joint stiffness matrix $K_{J}$ can be determined by 


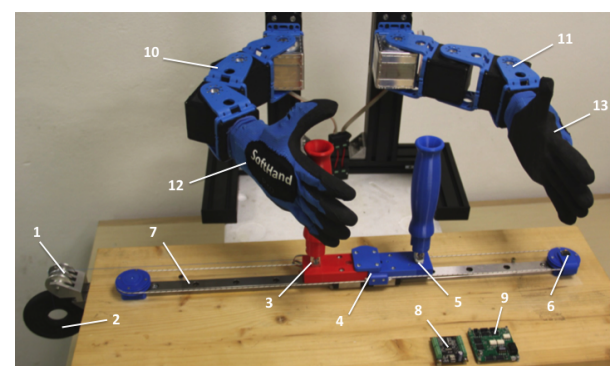

Fig. 7. The experimental setup for the handover task.

solving the optimization problem

$$
\min _{K_{J}}\left\|\bar{K}_{C}\left(q, K_{J}\right)-\hat{K}_{C}\right\|_{F}^{G},
$$

where $\|A\|_{F}^{G}:=\left\{\sum_{i, j}^{n} g_{i, j}\left|a_{i, j}\right|^{2}\right\}^{\frac{1}{2}}$ is the weighted Frobenius norm of $\|A\|$ w.r.t. a matrix of positive scalars $G$, and $\bar{K}_{C}\left(q, K_{J}\right)=\left(J(q)^{T} K_{C} J(q)\right)^{-1}$ is the Cartesian stiffness when the Cartesian displacement $\Delta_{x}$ is negligible. During the system evolution the joint stiffness is kept constant. We hence select the constant joint stiffness that solve the problem (11) along the whole trajectory. As reported in [1], the solution to the problem (11) for a single configuration can be obtained by solving a linear algebraic system of equations. Hence, the joint stiffness along the trajectory has been computed solving a least-square problem.

\section{EXPERIMENTAL RESULTS}

A comparison between three configurations, using different stiffness set, to perform the handover task are at the basis of experimental validation of the theoretical and simulation results obtained in the previous sections. The forces generated on the hands and the object displacement experimentally measured have been qualitatively compared with those produced in the simulations.

In order to ensure that the two hands perform the task in a more similar fashion to that of the carts in the physical model of Fig. 3 and due to the limits on the linear guide, the constraints on the maximum mass displacement are replaced by the constraints on the displacement of the two hands (fixed to $0.12[\mathrm{~m}]$ ). Furthermore, the maximum velocity of the end-effectors is set to $0.15[\mathrm{~m} / \mathrm{s}]$ and the uncertainty $\varepsilon$ to $0.02[m]$. With this set-up, the numerical solution of the optimization problem shows that the pair of stiffnesses that allows to accomplish the task minimizing the contact forces is $k_{l}=153.16[N / m], k_{r}=500[N / m]$. The trend of the forces obtained from the simulation results shows a good match with the acquired experimental data (see Fig. 8(a), 9(a), 10(a)): at the beginning of each experiment, due to the uncertainty effects, the right hand is subject to a force greater than the external one. When the task is completed, i.e. the load is completely transferred from the right to the left hand, the latter only needs to counteract the applied force.

The computed optimal stiffness set allows to limit the forces acting on the hands with respect to the Stiff/Stiff case that presents a peak of approximately $12[N]$. Moreover, the time required to accomplish the task is always smaller than the other cases: about $0.4[s]$, instead of $0.9[s]$ and $0.7[s]$ for the Soft/Soft and Stiff/Stiff case respectively.

The comparison of the object position measurements with the simulative trend shows some differences. Note that the oscillatory behavior expected in simulation is not measured by the sensors for the Stiff/Stiff case (see Fig. 10(b)). In the Soft/Soft case (see Fig. 9(b)) experimental data show that the object displacement at the end of the task is not zero as expected. In authors' opinion these are effects of the not modeled static friction of the system that in the stiff case damps the oscillations while in the soft case acts as a disturbance causing a steady-state error. These effects are visible also in the optimal case (see Fig. 8(b)).

\section{CONCLUSIONS}

The main contribution of this paper is the proof, through robust optimization, that under uncertainties it exists an optimal compliance choice that can improve the interaction performances as sustained by the related literature. A solution for the handover task, in terms of cartesian stiffness and reference signals, is theoretically and experimentally provided. It has been shown that the robust optimal control problem, under specific assumptions, can be recast into two deterministic linear programming problems. The optimal solutions have been provided varying the stiffness of the interacting entities of the case study in order to find the stiffness set which allow the minimization of the exchanged forces. Results show that in case of perfect knowledge of the framework, the optimal manipulator stiffness is high. On the other hand, in case of uncertainties a more compliant behavior has to be set. In particular the manipulator which holds the load to be exchanged has a higher value of stiffness than the approaching one: the optimal stiffness solution is hence stiff/soft. Moreover, an analogous optimal stiffness solution is obtained if the minimization of the number of steps necessary to accomplish the task is considered. The application of the non optimal stiffness configuration, e.g. stiff/stiff and soft/soft, does not provide neither the minimization of the exchanged forces nor the minimization of the number of steps to obtain a feasible solution. It is worth to note that results show a relationship between the uncertainty in the scenario and the optimal stiffness of the approaching manipulator, i.e. the more uncertain the scenario is, the more compliant the manipulator has to be. Simulation results have been validated experimentally and a qualitative comparison of the obtained forces has been provided w.r.t. the simulation cases.

\section{REFERENCES}

[1] A. Albu-Schäffer, O. Eiberger, M. Grebenstein, S. Haddadin, C. Ott, T. Wimböck, S. Wolf, and G. Hirzinger, "Soft robotics," Robotics \& Automation Magazine, IEEE, vol. 15, no. 3, pp. 20-30, 2008.

[2] G. Pratt, M. M. Williamson et al., "Series elastic actuators," in Intelligent Robots and Systems 95.'Human Robot Interaction and Cooperative Robots', Proceedings. 1995 IEEE/RSJ International Conference on, vol. 1. IEEE, 1995, pp. 399-406. 


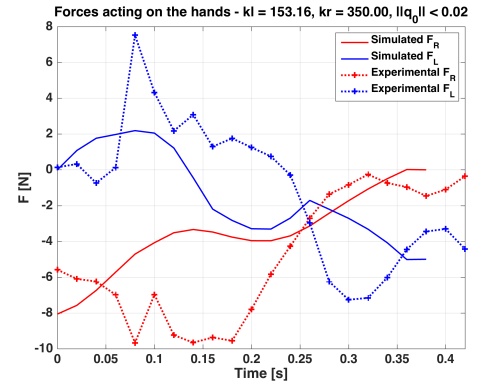

(a)

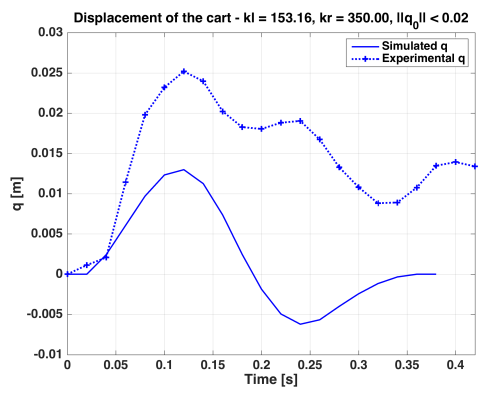

(b)

Fig. 8. Both plots depict the signal resulting from the experiments (red and blue dotted lines) with respect to those obtained in simulation (red and blue solid line), for the pair of optimal stiffnesses. In 8(a) there are the forces acting on the hands, while 8(b) shows the cart displacement.

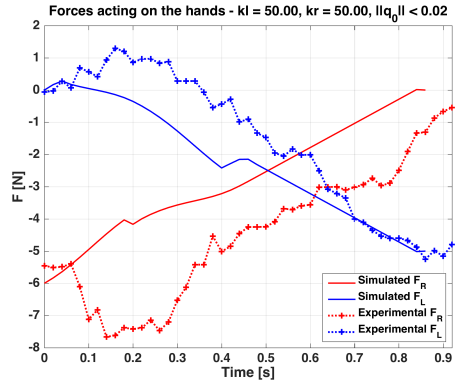

(a)

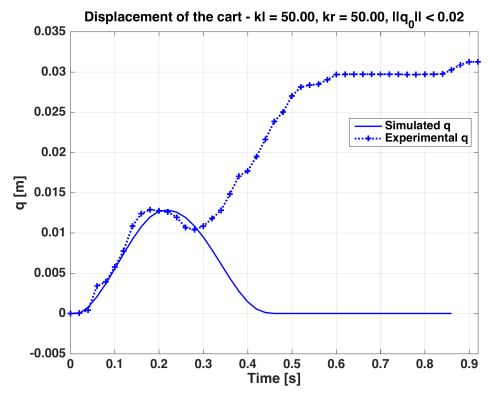

(b)

Fig. 9. Both plots depict the signal resulting from the experiments (red and blue dotted lines) with respect to those obtained in simulation (red and blue solid line), for the pair of Soft/Soft stiffnesses. In 9(a) there are the forces acting on the hands, while 9(b) shows the cart displacement..

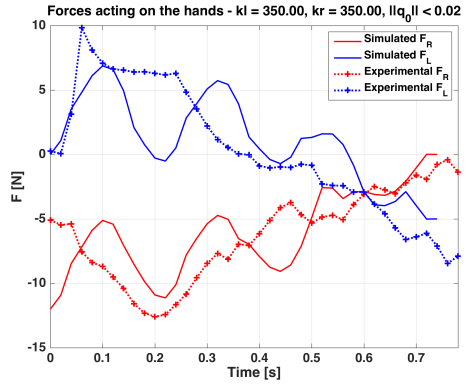

(a)

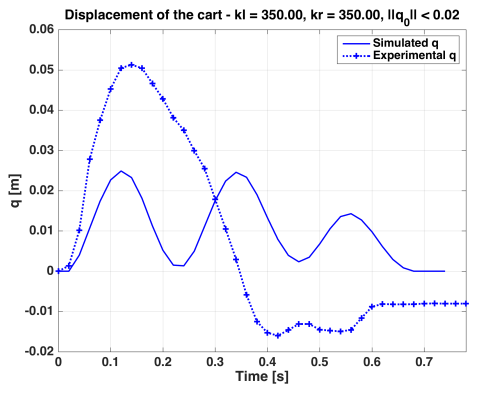

(b)

Fig. 10. Both plots depict the signal resulting from the experiments (red and blue dotted lines) with respect to those obtained in simulation (red and blue solid line), for the pair of Stiff/Stiff stiffnesses. In 10(a) there are the forces acting on the hands, while 10(b) shows the cart displacement.
[3] B. Vanderborght, A. Albu-Schäffer, A. Bicchi, E. Burdet, D. G. Caldell, R. Carloni, M. Catalano, O. Eiberger, W. Friedl, G. Ganesh et al., "Variable impedance actuators: A review," Robotics and Autonomous Systems, vol. 61, no. 12, pp. 1601-1614, 2013.

[4] F. Negrello, G. M., C. M. G., J. Malzahn, D. G. Caldwell, A. Bicchi, and N. G. Tsagarakis, "A modular compliant actuator for emerging high performance and fall-resilient humanoids," in HUMANOIDS2015, in 15th IEEE RAS Humanoids Conference, 2015.

[5] A. Bicchi and G. Tonietti, "Fast and soft arm tactics: dealing with the safety-performance tradeoff in manipulators design and control," IEEE Robotics and Automation Magazine, Special Issue on Safety Among Us, vol. 11, pp. 23-24, 2004.

[6] A. Velasco, G. M. Gasparri, M. Garabini, L. Malagia, P. Salaris, and A. Bicchi, "Soft-actuators in cyclic motion: Analytical optimization of stiffness and pre-load," in Humanoid Robots (Humanoids), 2013 13th IEEE-RAS International Conference on. IEEE, 2013, pp. 354-361.

[7] M. Uemura and S. Kawamura, "Resonance-based motion control method for multi-joint robot through combining stiffness adaptation and iterative learning control," in Robotics and Automation, 2009. ICRA'09. IEEE International Conference on. IEEE, 2009.

[8] S. Haddadin, M. Weis, S. Wolf, and A. Albu-Schäffer, "Optimal control for maximizing link velocity of robotic variable stiffness joints," in IFAC world congress, 2011, pp. 6863-6871.

[9] M. Garabini, A. Passaglia, F. Belo, P. Salaris, and A. Bicchi, "Optimality principles in variable stiffness control: The vsa hammer," in Intelligent Robots and Systems (IROS), 2011 IEEE/RSJ International Conference on. IEEE.

[10] N. Hogan, "Impedance control: An approach to manipulation," in American Control Conference, 1984. IEEE, 1984, pp. 304-313.

[11] M. Jafarian, G. van Oort, R. Carloni, and S. Stramigioli, "Performance comparison of a planar bipedal robot with rigid and compliant legs," in Proceedings of the 18th IFAC World Congress, 2011, pp. 6924-6929.

[12] D. Bertsimas, D. B. Brown, and C. Caramanis, "Theory and applications of robust optimization," SIAM review, vol. 53, pp. 464-501, 2011.

[13] Q. Zhang, M. F. Morari, I. E. Grossmann, A. Sundaramoorthy, and
J. M. Pinto, "An adjustable robust optimization approach to scheduling of continuous industrial processes providing interruptible load," Computers \& Chemical Engineering, vol. 86, pp. 106-119, 2016.

[14] A. Lorca and X. A. Sun, "Adaptive robust optimization with dynamic uncertainty sets for multi-period economic dispatch under significant wind," IEEE Transactions on Power Systems, vol. 30, no. 4, pp. 17021713, 2015.

[15] K. Debrabant, N. Kr et al., "Robust optimization of robotic pick and place operations for deformable objects through simulation," in 2016 IEEE International Conference on Robotics and Automation (ICRA). IEEE, pp. 3863-3870.

[16] J. Luo and K. Hauser, "Robust trajectory optimization under frictional contact with iterative learning," in Robotics, Science and Systems (RSS), 2015.

[17] Catalano et al., "Vsa-cubebot: A modular variable stiffness platform for multiple degrees of freedom robots," in Robotics and Automation (ICRA), IEEE International Conference on. IEEE, 2011, pp. 50905095.

[18] B. Siciliano, L. Sciavicco, L. Villani, and G. Oriolo, Robotics: modelling, planning and control. Springer Science \& Business Media, 2010.

[19] W. P. Chan, C. A. Parker, H. M. Van der Loos, and E. A. Croft, "A human-inspired object handover controller," The International Journal of Robotics Research, vol. 32, no. 8, pp. 971-983, 2013.

[20] Natural machine motion initiative. [Online]. Available: http://www. naturalmachinemotioninitiative.com/

[21] M. G. Catalano, G. Grioli, E. Farnioli, A. Serio, C. Piazza, and A. Bicchi, "Adaptive synergies for the design and control of the pisaiit softhand," International Journal of Robotics Research, vol. 33, pp. 768-782, 2014.

[22] A. Albu-Schaffer, M. Fischer, G. Schreiber, F. Schoeppe, and G. Hirzinger, "Soft robotics: what cartesian stiffness can obtain with passively compliant, uncoupled joints?" in Intelligent Robots and Systems, 2004.(IROS 2004). Proceedings. 2004 IEEE/RSJ International Conference on, vol. 4. IEEE, pp. 3295-3301. 